the main cause of the electrification in both layers is the ultra-violet light of the sun, and in magnetically quiet conditions (no magnetic storms) this electrification is actually less in the arctic regions than in England. While the normal undisturbed conditions fit in with the ultra-violet light theory, the conditions during magnetic disturbances and auroral displays can only be satisfactorily explained on the assumption that the electrification spreads to abnormally low levels because of the influx of particles from outside the earth's atmosphere. Experiments arranged by Mr. R. A. Watson Watt in the Library of the Royal Institution after the lecture well illustrated the behaviour of these incoming particles when influenced by the magnetism of the earth. Streams of electrons in a vacuum tube striking a magnetic model of the earth near the poles avoid the tropics, being caught up and deflected by the earth's magnetic field. The very complicated paths followed by the electrons -often looping back on themselves-realistically imitated auroral displays. The actual receiver used by the Arctic Circle expedition was shown in operation in measuring the height of the reflecting layers over London by using pulses of energy emitted from King's College, London.

\section{The British Grid and Underground Distributing Cables}

Mr. P. V. Hunter, in his presidential address to the Institution of Electrical Engineers on October 26, said that during the last ten years the electric supply of Great Britain has more than doubled. It seems to be increasing at a practically constant rate. The small setbacks that occurred in the growth of supply were quite definitely due to political uncertainty at those periods, but were more than counterbalanced by the 'boom' year of 1929. The progress of the 'grid' is quite up to expectations and the country will soon be greatly benefited by it. Uncertainty still exists as to whether to use 11,000 volt underground cables or overhead wires for rural electrification from the grid. Mr. Hunter said that in many cases it would be advisable to run cables across country. In this case it is much less costly to maintain them than when they are laid in public roads. A special type of rural cable for 11 kilovolt working could be standardised. If this were done, it would be much cheaper to manufacture and would result in economies in many directions. In Holland, rural areas have been developed by 10,000 volt underground cables on a vast scale, more than 10,000 miles of such cable having been already laid. The network covers practically the whole country. So far as freedom from interruption and voltage variation is concermed, the supply is most satisfactory and the prices charged for electricity in rural districts are quite reasonable. The annual cost of repairs is less than 0.5 per cent of the original capital cost and is not a growing percentage. This is most satisfactory, as much of the cable is more than ten years old and, compared with modern practice, the sheaths are poorly protected against corrosion. The Dutch are convinced that underground cables are a great success and will undoubtedly continue to use them.
In conclusion, Mr. Hunter gave an instructive résumé of the vast amount of scientific work done in connexion with very high voltage cables.

\section{New Lamp for Street Lighting}

A VERY interesting new type of lamp, called the Osira, has been developed in the G.E.C. Research Laboratories at Wembley. It is intended for use in lighting stretches of arterial and country roads where the illumination required is not high and so the lack of good colour discrimination is of little importance. Blues, greens and yellows appear as in daylight, but red colours appear brownish. The lamp takes 400 watts and has an efficiency more than double that of a corresponding gas-filled tungsten lamp taking the same power. In the G.E.C. Journal for November, Mr. J. W. Ryde gives a technical description of the lamp. It consists of two cylindrical bulbs one inside the other. The inner bulb contains rarefied gases at low pressure and a trace of mercury. The space between the bulbs is vacuous. The electric discharge takes place between electrodes sealed in each end of the inner bulb. The electrodes consist of small sticks of alkaline earth oxides which have been heated to a high temperature during the process of manufacture. This is done by passing a current through spirals of wire surrounding the electrodes. They are then connected with an ordinary screw cap holder. When the voltage is applied, a discharge takes place through the rarefied gases in the volume of the inner bulb. As this bulb warms up, the mercury is volatilised. The discharge then begins to contract and finally takes the form of a narrow column, six inches long, extending between the electrodes. The electrically excited atoms in this column emit a brilliant light and are the counterpart of the filament in the glow lamp.

THe new lamp lights up automatically when switched on, no devices are required for starting the discharge and no filament transformers are required for heating the electrodes. It is connected directly to the alternating current supply mains in series with a choking coil. A condenser is sometimes placed across the mains so as to raise the 'power factor' of the load, but this does not affect the performance of the lamp itself. In this type of lamp, the current is carried by ions and its effective resistance depends on their number. The ions, which are negatively charged electrons and positively charged atoms, are formed by the discharge itself at a rate which increases with the current. They re-combine with extreme rapidity and so again form normal atoms. The resistance of the bulb at any moment depends on the current passing. The lamp resistance therefore does not remain constant when the voltage is changed. In a similar way the resistance of an ordinary in. candescent lamp changes with its temperature. If there were no choke coil present, the current would rise to a very high value and the lamp would break down. A change of one per cent in the supply voltage corresponds to a $2 \cdot 6$ per cent change in the power taken and to a $3 \cdot 5$ per cent change in the light. In the gas-filled tungsten lamp, the corresponding 